\title{
Prediction of Protein Interactions by the Domain and Sub-cellular Localization Information ${ }^{\text {II }}$
}

\author{
Jinsun Hong and Kyungsook Han ${ }^{*}$ \\ School of Computer Science and Engineering, Inha University, Inchon 402-751, Korea \\ khan@inha.ac.kr
}

\begin{abstract}
There has been a recent interest in the computational methods for predicting genome-wide protein interactions due to the availability of genome sequences of several species, the difficulty of detecting whole protein interactions in higher species even with the current high-throughput experimental methods, and the common perception of the data generated by high-throughput experimental methods as noisy data. However, data predicted by computational methods as well as that detected by high-throughput experimental methods inherently contain extremely many false positives. Several methods have been developed for estimating the reliability of experimental protein interaction data, but there are few for predicted interaction data. This paper presents a prediction method of protein-protein interactions using the protein domain and sub-cellular localization information, and experimental results of the method to the protein-protein interactions in human.
\end{abstract}

\section{Introduction}

An intrinsic problem with high-throughput methods for detecting protein-protein interactions is that data generated by the methods are extremely noisy, even more so than is the case for gene expression data, so one cannot simply use the data blindly [1]. More than half of current high-throughput data are estimated to be spurious [2]. Although it is possible to focus on interactions with higher reliability using only those supported by two or more sources of evidence, this approach invariably throws out the majority of available data [1].

In an attempt to improve the reliability of interaction data, we have recently generated a new set of interaction data using protein domain [3-5] and sub-cellular localization. This paper presents a scoring scheme for assessing the reliability of the protein interaction data predicted by a computational method called homologous interactions, and experimental results of the scheme to the protein-protein interactions in human. The scoring method is more general than a typical method of selecting cofunctional or co-localized protein pairs only since some pairs of proteins interact more frequently than others despite their different functions or locations in a cell. The protein-protein interaction data predicted by the method was compared to the

\footnotetext{
II This work was supported by the Ministry of Information and Communication of Korea under grant IMT2000-C3-4 and by KOSEF through the Systems Bio-Dynamics Research Center.

* To whom correspondence should be addressed.
} 
experimental data in HPRD (http://www.hprd.org/), and the results show that the method predicts reliable protein-protein interactions and is useful for assessing the reliability of protein-protein interactions.

\section{Prediction Methods and Experimental Results}

The prediction system of protein-protein interactions is composed of two parts: predicting protein interactions and assessing the reliability of the predicted interactions (Fig. 1).

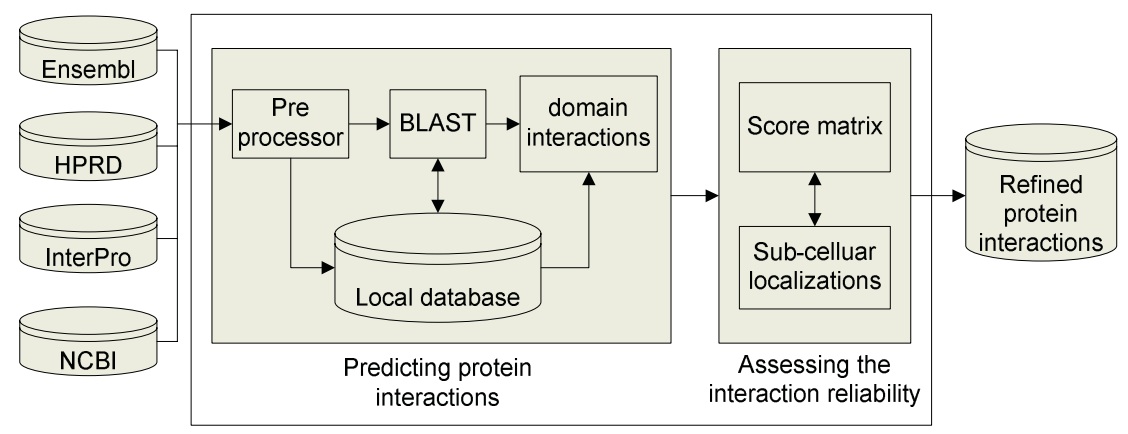

Fig. 1. Architecture of the prediction system of protein-protein interactions

The interaction prediction part extracts relevant data (for example, protein names and sequences, sub-cellular localizations, domains and their amino acid sequences, and known protein-protein interactions) from Ensembl (http://www.ensembl.org/), HPRD (http://www.hprd.org/), InterPro (http://www.ebi.ac.uk/interpro/), and NCBI (http://www.ncbi.nih.gov/), and removes redundant data. Suppose a protein $\mathrm{X}$ with domains $\mathrm{a}, \mathrm{b}$, and $\mathrm{c}$ and protein $\mathrm{Y}$ with domains $\mathrm{d}$ and $\mathrm{e}$. When proteins $\mathrm{X}$ and $\mathrm{Y}$ interact each other, we can predict 6 domain-domain interactions of $(a, d),(b, d),(c$, d), $(a, e),(b, e),(c, e)$, which are in turn used to predict new protein-protein interactions. PSI-BLAST [6] is used to identify domains from protein sequences.

The reliability assessing part constructs a score matrix from the interaction frequency at a specified sub-cellular localization, and the score matrix is used to evaluate the reliability of predicted protein-protein interactions. The information on protein, protein-protein interactions, and sub-cellular localization was obtained from HPRD that has human protein-protein interactions with the sub-cellular localization information. The sub-cellular localization schema of MIPS (http:/www.mips.gsf.de/) was used to classify 20 sub-cellular localizations in human.

The sub-cellular localization score $\left(S_{l}\right)$ was computed using equation 1 , in which $I_{l}\left(p_{i}, p_{j}\right)$ is the number of interactions between proteins $p_{i}$ and $p_{j}$ in a same compartment $l$, and $N$ is the number of proteins that participate in protein-protein interactions. Interactions with the sub-cellular localization score $S_{l}=0$ are the cases in which source or target protein has no sub-cellular localization information. 


$$
S_{l}\left(p_{i}, p_{j}\right)=\frac{1}{N} \sum I_{l}\left(p_{i}, p_{j}\right)
$$

We constructed 2,272 domain-domain interactions between 1,712 human domains and predicted 5,188 protein-protein interactions between 4,205 human proteins. In the reliability assessing part, localization ID from 1 to 20 was assigned to 5,188 proteinprotein interactions and the value of a score matrix was applied to these interactions. 3,839 protein-protein interactions have higher value than 0 and others have value of 0 .

We compared the predicted data by our system to the experimental data in HPRD. Fig. 2 shows $S_{l}$ scores in the range from 0 to 2 for the two data sets. A vertical axis presents an accumulated percentage at each score. In the experimental data of HPRD, there were 1,336 protein-protein interactions $(37 \%)$ of 3,611 protein-protein interactions with $S_{l}=0$. In the predicted data by our system, there were 1,349 proteinprotein interactions $(26 \%)$ of 5,188 protein-protein interactions with $S_{l}=0$. This indicates that the prediction system generates more reliable interactions than the experimental data of HPRD.

Fig. 3 shows the sub-cellular localization distribution that includes cytoplasm, extra-cellular, membrane, nucleus between existing experimental 1,179 interactions obtained from BIND database and 5,188 interactions predicted by our system. In general, our system contains more protein interactions than existing experimental data in most cell compartments, and much more protein interactions in nucleus.
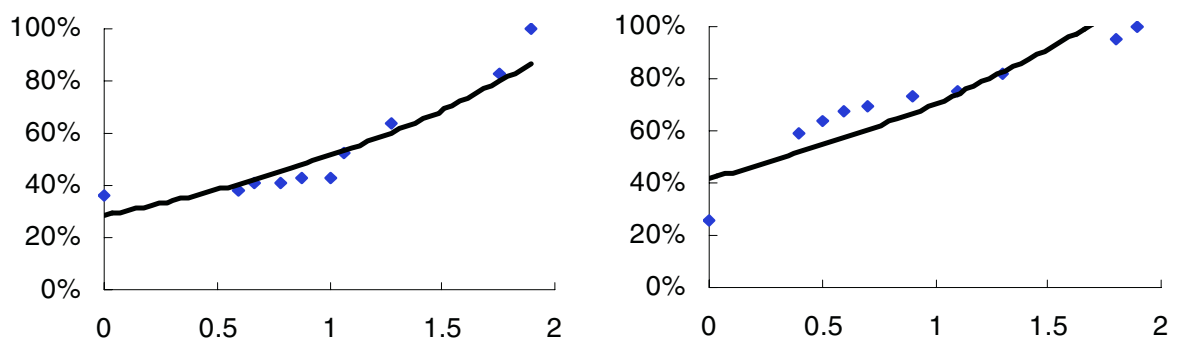

Fig. 2. $S_{l}$ scores of the HPRD data (left) and those of our system (right)
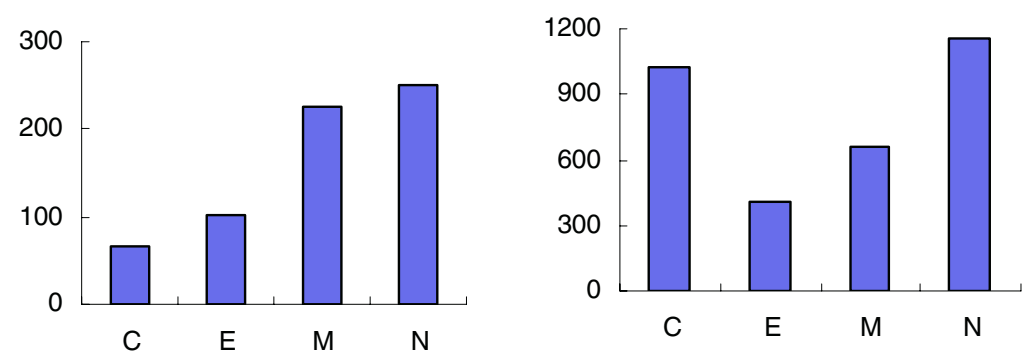

Fig. 3. The sub-cellular localization distribution of the BIND data (left) and our data (right). C: Cytoplasm, E: Extra-cellular, M: Membrane, N: Nucleus 
We also computed the Interaction Generality (IG) values [7] to assess the reliability of protein-protein interactions. The IG value of an interacting protein pairs A-B is defined as the number of proteins directly interacting with protein A or B. Proteins with IG value less than 5 were $76 \%$ of proteins that participates in 3,839 predicted proteins-protein interactions.

\section{Conclusions}

This paper presented an intuitive, computationally feasible scoring scheme for measuring the reliability of a large-scale data set of protein-protein interactions, and for filtering potentially false positives in the data set. The number of potential artifacts can be substantially reduced be removing data with low score from the data set. Since the reliability score is computed for individual protein interactions in a data set, and the score can be directly used to filter out spurious interactions (that is, those interactions with low scores) from the data set. The overall reliability of the entire data set can also be computed by taking the average scores of the interactions in the data set. In this study we computed sub-cellular localization scores only, but the scoring scheme is general enough to incorporate any number of subscores depending on the sources of information available.

\section{References}

1. D'haeseleer, P., Church, G.: Estimating and improving protein interaction error rates. Proceedings of the Computational Systems Bioinformatics Conference (2004) 208- 215

2. von Mering, C, Krause, R., Snel, B., Cornell, M., Oliver, S.G., Fields, S., Bork, P.: Comparative assessment of large-scale data sets of protein-protein interactions. Nature 417 (2002) 399-403

3. Antonina, A., Dave, H., Steven, E.B., Tim, J. P.H., Cyrus, C., Alexey, G. M.: SCOP database in 2004: refinements integrate structure and sequence family data. Nucl. Acids. Res. 32 (2004) D226-D229

4. Einat, S., Hanah, M.: Correlated Sequence-signatures as Markers of Protein-Protein Interaction. J. Mol. Biol. 311 (2001) 681-692

5. Lappe, M., Park, J., Niggemann, O., Holm, L.: Generating protein interaction maps from incomplete data: application to fold assignment. Bioinformatics 17(2001) 149-156

6. Altschul, S.F., Madden, T.L., Schffer, A.A., Zhang, J., Zhang, Z., Miller, W., and Lipman, D.J.: Gapped BLAST and PSI-BLAST: a new generation of protein database search programs. Nucleic Acids Res. 25 (1997) 3389-3402

7. Saito, R., Suzuki, H., Hayashizaki., Y.: Interaction generality, a measurement to assess the reliability of a protein-protein interaction. Nucleic Acids Res. 30 (2002) 1163-1168 